\title{
Analysis and synthesis parameters influencing to the effects of impact
}

\author{
Peter Šulka ${ }^{1, *}$, Alžbeta Sapietová ${ }^{1}$, Vladimir Dekýšs ${ }^{1}$, Milan Sapieta ${ }^{1}$ \\ ${ }^{1}$ Department of Applied Mechanics, Faculty of mechanical engineering, University of Žilina, \\ Univerzitná 8215/1, 01026 Žilina, Slovak Republic
}

\begin{abstract}
In the solutions will be presented to the interaction of two bodies loaded with impact effects in the environment of software MSC. ADAMS. By performing a sensitivity analysis of the parameters influencing to the effects of impact and function parameters of impact will be obtained parameters for optimum setting MSC.ADAMS for acquirement feedback, as the most identical with reality.
\end{abstract}

Keywords: Analysis, impact, compression, restitution, MSC.ADAMS

\section{Introduction}

Analysis of a wide range of problems in technical practice uses mathematical representation of the future product in a virtual computerized form. Such modelling of the problem requires that the parameters input to the solution, and also the outputs, be consistent with reality.

Keeping this objective in mind, this paper deals with dynamic analysis of load by forces - in this case the so-called impact forces - that cause destruction of the device. If there is interaction between two bodies and the bodies do not move relative to each other, we talk about their contact. Interaction of bodies that move relative to each other is called impact. Impact is characterized by short-term significant forces occurring in the contact area. These impact forces act for a very short time - in the order of milliseconds. Despite the above, these forces cause significant changes in the velocity and thus in the momentum of the participating bodies. The change is by a final value. Therefore, each body must receive a final impulse equivalent to the change in its momentum. However, the impact forces must be very large in order to generate the final impulse in a very short time. This is then followed by the above-mentioned destruction of the material when the forces act. A better understanding of the interdependence between the impact force and the mechanism parameters allows engineers to find more efficient and innovative solutions. Therefore, we carried out analysis of the impact force in the tensioning mechanism using the IMPACT function in the MSC.ADAMS software.

\footnotetext{
* Corresponding author: peter.sulka@fstroj.uniza.sk

Reviewers: Tomáš Lack, Milan Nad'
} 


\section{Theoretical background of contact forces in the MSC.ADAMS software environment}

When the bodies in the virtual prototype models are in contact with each other, the CONTACT function allows making or modifying the contact in the ADAMS/View software environment. Bodies in the contact models can touch each other at points, touch points can travel along pre-described curves, or the curves of one body can be in contact with the curves upon the other body. We can also define the contact between nondeformable bodies at a standstill and in motion. We can model the contact of bodies with stiff (rigid) as well as flexible elements [1].

Solver algorithms detect the contacts of two geometric objects, determine the touch point position, calculate the position of the common normal at the touch point, and calculate the normal and slipping relative velocity of the point in contact.

In terms of dynamics, it is necessary to distinguish:

- discontinuous (pulse) contacts occurring in short periods of time - impacts, for which ADAMS/Solver creates an estimate of the contact force by modeling the course of local deformation. The damping force with the corresponding damping coefficient or restitution coefficient represents loss of energy during the collision. In the first phase, compression occurs during which kinetic energy is converted to potential and dissipative energy of the compressed material. In the second phase, the accumulated potential energy causes reverse motion accompanied by the transformation of potential energy into kinetic and again dissipative energy.

- continuous contacts represented by a model in the form of a non-linear spring-damper system in which stiffness takes into account elasticity of contacting surfaces and damping, in turn, takes into account energy dissipation, and the bodies are not separated after the collision. Calculation of contact forces is carried out separately for each point, and the resulting effect is their sum.

The force acting in the contact point is calculated either by the restitution method (POISSON model) or by the IMPACT function. Both contact force models result from the regulation of normal contact constraint penalty, where the constraint in this modelling technique is secured mathematically by applying forces according to the constraint gradient, and the magnitude of forces is a function of the constraint breach level [2, 3].

Theoretically, there is no mutual penetration of surfaces at the contact of nondeformable bodies, which can be expressed in the form of inequalities for unilateral constrain. Additional constrain conditions can be expressed also by introducing Lagrange coefficients, but the penalty regulation is advantageous due to its simplicity since no additional equations or variables are needed. The advantage is the ability to apply algorithms of active and inactive conditions for unidirectional constraint as well as for clear interpretation from the physical point of view. The disadvantage of the penalty regulation is that the user is responsible for setting a suitable penalty parameter in the form of material stiffness, a high value of which may cause difficulties in the work of solver integration algorithms. Mutual penetration of the body surfaces is monitored by the gap function $g$, whose positive value indicates penetration. A positive value of the normal contact force in turn means that it is trying to separate the bodies, and its value is non-zero while the surfaces are in contact [4].

The impact force model in MSC.ADAMS is implemented using the IMPACT function. An example of modelling using the IMPACT function is a ball falling towards the ground, plotted at the beginning of the investigated motion and at impact (Fig. 1). If the distance between the $I$ and $J$ markers reaches $x_{l}$, the impact force IMPACT turns on. This occurs when two bodies collide. As long as the distance between the $I$ and $J$ markers is greater than $x_{1}$; the force is zero. 


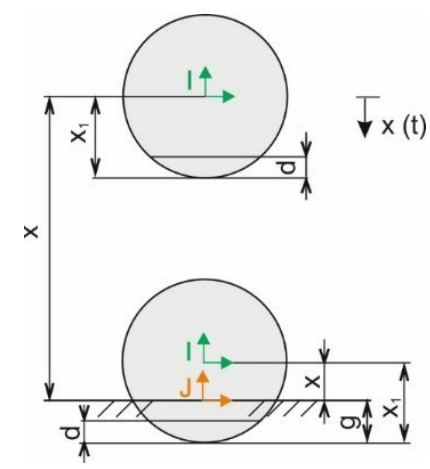

Fig. 1. Example illustrating the IMPACT function

The impact force has two components, a spring or stiffness component, and a damping or viscous component. The stiffness component is proportional to the stiffness coefficient $k$, that is load per unit length, i.e. it is a function of changes in the distance between the $I$ a $J$ markers. The stiffness component opposes the mutual penetration of the body surfaces. The damping coefficient $c$ belonging to the damping component of the force is a function of the velocity of mutual penetration of the body surfaces. The damping opposes the direction of relative motion. The damping coefficient achieves a maximum value $c_{\max }$, at a user-defined penetration $d$ (Fig. 2b).

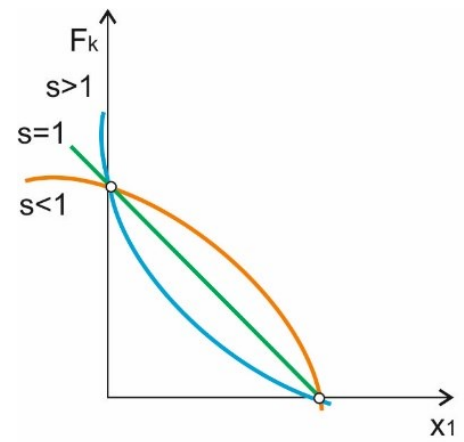

a)

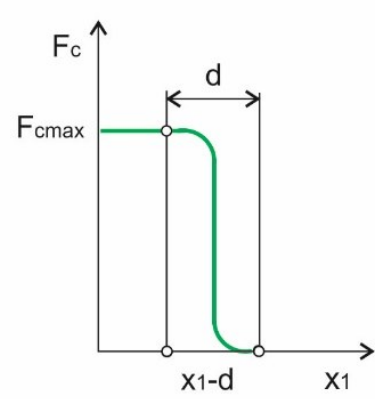

b)

Fig. 2. a) Exponent $s$ of the force in a non-linear spring, b) Depth $d$ of the penetration to the maximum damping force

Figure 3 shows a local coordinate frame of the $I$ marker that represents the first body and a local coordinate frame of the $J$ marker that represents the second body. The impact force model consists of the force acting in the compression spring, where $F_{k}=k x$, and of the force acting in the damper, where $F_{c}=c \dot{x}$. Then the impact force magnitude is Fimpact $=F_{k}+F_{c}=k x-c \dot{x}$. Note that the IMPACT function solution in the MSC.ADAMS software environment results in a real number $[5,6]$. 


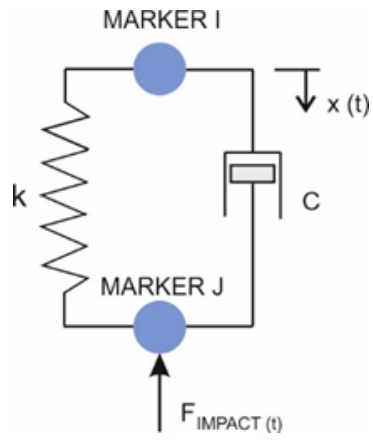

Fig. 3. Model of unilateral impact force by the IMPACT function

Given the solver problems with high stiffness values and the need to include critical material properties into the impact model, the solver algorithm works with an extended mathematical model that includes non-linear viscous damping dependent on the magnitude and velocity of mutual penetration of surfaces.

The equation defining IMPACT is:

$$
\operatorname{IMPACT}=\left\{\begin{array}{cc}
\operatorname{Max}\left(0, k\left(x_{1}-x\right)^{s}-\operatorname{STEP}\left(x, x_{1}-d, c_{\max }, x_{1}, 0\right) . \dot{x}\right) & : x<x_{1} \\
0 & : x \geq x_{1}
\end{array}\right\},
$$

Based on the presented impact model, the following applies (Fig. 1):

$x>x_{1}$, no mutual surface penetration occurs and the force is zero (penetration $g=0$ )

$x<x_{1}$, penetration $\mathrm{g}$ occurs at the end closer to the $J$ marker, and the force is $>0$.

Also note that when $g<d$, the instantaneous damping coefficient is a cubic step function of the penetration $g$.

When $g>d$, the instantaneous damping coefficient is $c_{\max }$.

The MSC.ADAMS $(\mathrm{C}++)$ software solver never returns a negative force for impact. If the above expression is negative, the solver returns a force value of zero.

It must be said that in terms of theory there has been a failure so far to mathematically describe the phenomena during impact, although the impact models based on energy balance before and after the impact correlate the corresponding parameters with sufficient precision [7].

\section{Setting the parameters of the pulse contact in MSC.ADAMS}

In the following, we will focus on analysis and synthesis parameters for setting solutions intermittent (impulse) terminal i.e. IMPACT of MSC. ADAMS.

Fig. 4 displayed two bodies in software MSC.ADAMS, which are labeled 1 a 2. These bodies come together and initial difference in velocity. Ordinarily they first touch at a contact point $A$. During a very short time of contact, the point $A_{I}$ on the surface of the body 1 identical to point $A_{2}$ on the surface of the body 2 . When at least one of the bodies, 1 and 2 , a surface which is smooth topologically in the contact (i.e. surface with a continuous curvature), there is a plane tangent to this surface at point $A$. Identical contact points $A_{l}$ and $A_{2}$ are situated in this contact plane. If both bodies are convex and the surfaces have continuous curvature near the contact point, then this tangent plane is tangential to both surfaces that touch at point $A$, i.e. the surfaces of the colliding bodies have a common tangent plane. Moreover, unit vector $n$ specifies the direction of the normal to the tangent plane (Fig. 4). The contact force and changes in relative velocity at the contact point $A$ will be resolved into components normal and tangential to the common tangent plane. 


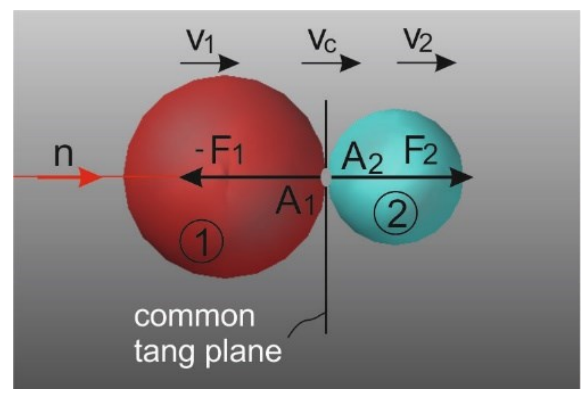

Fig. 4. Collinear impact of the two bodies

Weight of the opposing bodies 1 and 2 is $m_{l}=12 \mathrm{~kg}$ and $m_{2}=10 \mathrm{~kg}$, and the timedependent velocity at the start of movement is $v_{l}=10 \mathrm{~m} / \mathrm{s}$ and $v_{2}=6 \mathrm{~m} / \mathrm{s}$ pointing in the direction parallel to $n$. In a direct collision, these bodies are not rotating when they collide, so that velocity is uniform in each body (i.e. in each point of bodies). During contact, there are equal but opposite compressive reaction forces which develop at contact points $A_{l}$ and $A_{2}$. These forces oppose interference or overlap of the contact surfaces. In the case of direct impact between collinear bodies, the relative velocity between the contact points $A_{1}$ and $A_{2}$ remains parallel to the common normal direction throughout the contact period. A reaction force develops at the contact point because of compression of the local contact region.

This force opposes relative motion during contact. In a direct collision, the reaction force acts in the normal direction, i.e. parallel to the velocities, as illustrated in (Fig. 4). If the colliding bodies are hard, the contact force is very large in comparison with any body force, consequently, in rigid body impact theory anybody or applied contact forces of finite magnitude are negligibly small in comparison with the reaction at the contact point $A$. The finite body forces are ignorable because they do not work during the vanishingly small displacements that develop during an almost instantaneous collision. This is why a body force such as gravity does not affect the changes in velocity occurring in a collision. During impact between hard bodies, the only active forces are reactions at points of contact.

\subsection{Compression and restitution}

In the process of action of the contact force exist two phases and the compression and restitution. After the colliding bodies first touch, the contact force $F(t)$ rises as compression of bodies in contact point. We will consider with small elastic part of a total weight of which amounts to significant deformation of the contact point. If flexibility is rateindependent, the maximum indentation and maximum force occur simultaneously when the normal component of relative velocity vanishes $[8,9]$. (Fig. 5) illustrates the normal force as a time function.

Fig. 6 displays the separation of the contact period into an initial phase of approach or compression and subsequent phase of restitution. During compression, kinetic energy of relative motion is transformed into internal energy of deformation by the contact force with action of contact, force reduces the initial normal relative velocity of the colliding bodies while simultaneously an equal but opposite contact force does work that increases the internal deformation energy of the deformable particle $[6,8]$. 


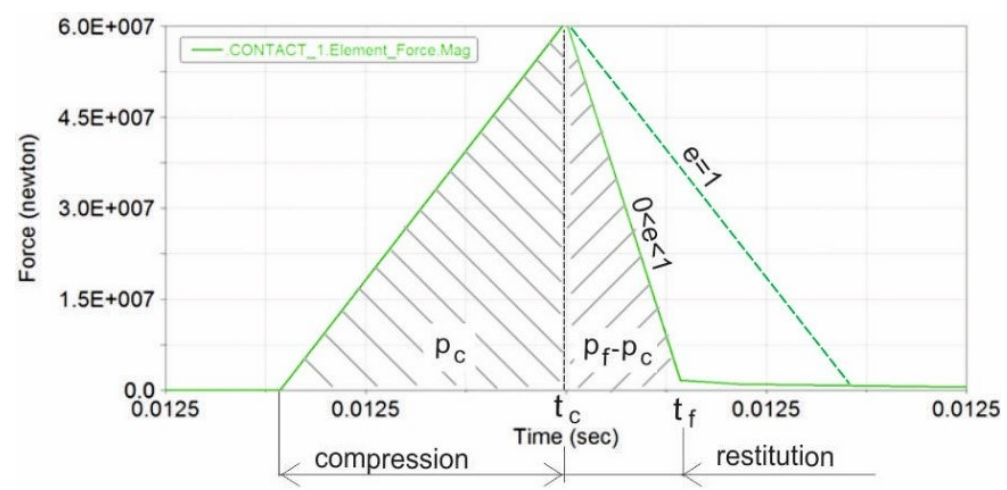

Fig. 5. Change of contact of the impact force as a function of time

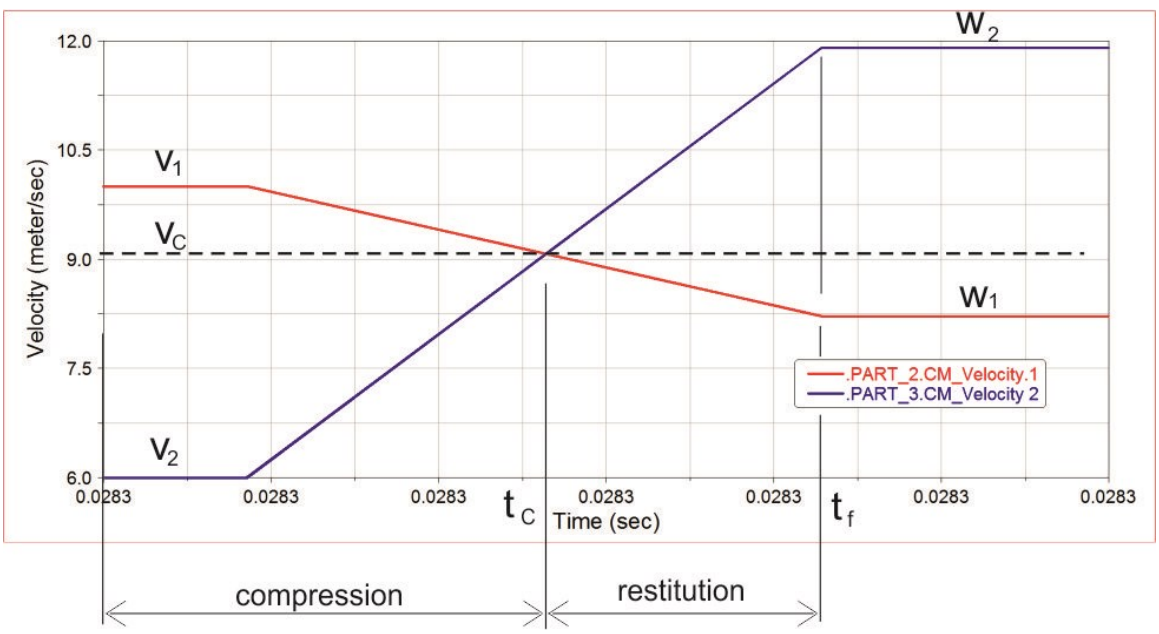

Fig. 6. Changing the normal velocities of the conflicting bodies as a function of time

The compression phase terminates and restitution begins when the normal relative velocity of the contact points vanishes. In a subsequent phase of restitution, internal elastic area drives the bodies apart. Elastic strain energy stored during compression generates the force that drives apart during restitution - the work done by this force restores part of the initial kinetic energy of relative motion. The compliance of the deforming region during restitution is smaller than that during compression $[2,9]$.

At any time $t$ after incidence, the normal component of contact force $F$ has and impulse $p c$, which equals the area under the curve of force, shown in (Fig. 5). During the compression of increasing monotonically pulse that slows down the body 1 , and increases the speed of the body 2 as shown in (Fig. 6). Consider the moment when changes in compression bruising to restitution as $t c$. Colliding elements have a relative speed between the contact points, which is lost at the end of compression:

$v(t c)=0$, i.e. ends when the contact points have the same speed $v c$ in the normal direction (Fig. 6). While $p f$ is finite impulse in compartments (Fig. 5). In (Fig. 6) is illustrated that each contact point of the body there is a change in velocity, which is directly proportional to the normal reaction of impulse in contact point $A$. The reaction impulse $p_{c}=\int_{0}^{t_{c}} F(t) d t$ that causes the bodies to move at the same speed is called normal pulse compression. This impulse is characteristic that is required for analysis of the collision process. 
By the direct impact finite impulse pf and the total kinetic energy absorbing directly related to the coefficient of restitution e. Restitution coefficient introduces the dissipation of (kinetic) energy due to inelastic deformation in the field of contact point. This factor has a value in the range $0 \leq e \leq 1$, whereas 0 means plastic deformation (i.e. without final separation, so that no part of initial kinetic energy of the normal relative motion is not restored) [5], while by the $e=1$ attends to the perfectly elastic collision (i.e. no loss of kinetic energy of the normal relative motion).

It is needed to say, that proportion of final to initial relative velocity of a normal pulse for the restitution of the pulse at compression are directly dependent on a valid equations:

$$
e=-\frac{v_{f}}{v_{0}}, e=-\frac{p_{f}-p_{c}}{p_{c}} .
$$

\subsection{Influence of step-size to calculate the impact size}

That the impact force was recorded, it is necessary to select the appropriate step size. To do this, we used sensitivity analysis during which we carry out a series of simulations to determine the impact of the integration step for calculating the contact force (Tab. 1). Further smoothing of the step-size was not performed, as the program did not converge.

Reaction forces are indefinitely large, nevertheless, they produce a finite impulse that continuously changes in relative velocity during the instant of contact.

Table 1. Step-size of iterative process

\begin{tabular}{|c|c|c|c|}
\hline Simulation & Contact 1 & Contact 2 & Contact 3 \\
\hline Step-size & 0.0002 & 0,00002 & 0,000002 \\
\hline
\end{tabular}

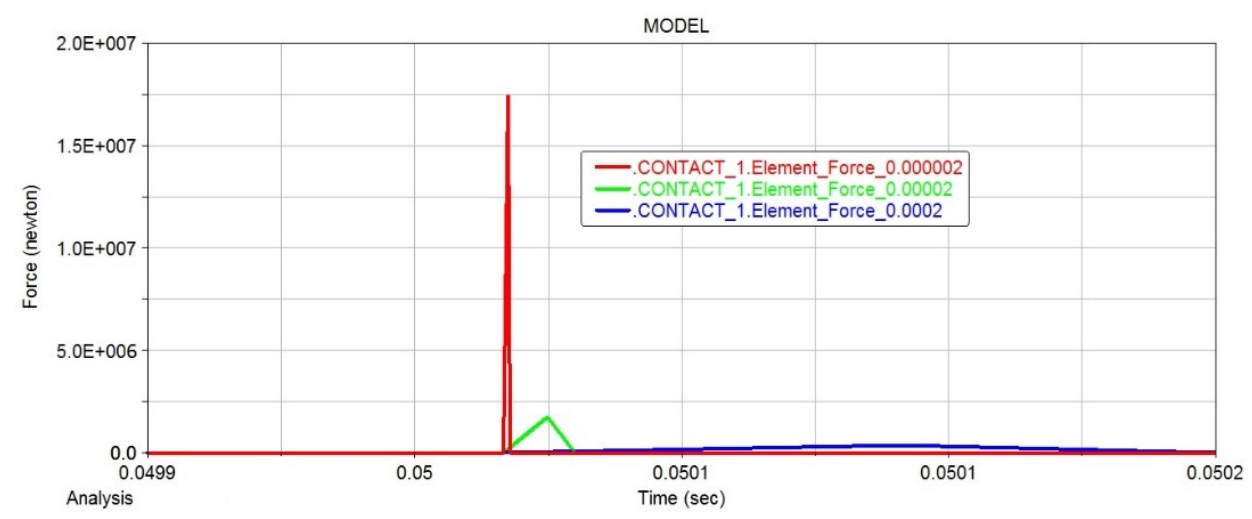

Fig. 7. Changing the step-size value regarding to the result's accuracy

From comparison of process in (Fig. 7) is illustrated, that the small integration step is justified only in the time interval in which the contact occurs. Otherwise, too small value of time step too unnecessarily slows down the simulation. Computational efficiency can be achieved by controlling the integration step simulation scripts where soften up over just before impact during collision and just after impact. 


\subsection{Influence of impact integrator and formulations DAE equations}

In order to achieve from the simulations of virtual prototype optimal results, was carried amount of computational simulations by entering various types of integrators (GSTIFF, WSTIFF, HASTIFF) and formulations of differential and algebraic equations (DAE, I3, SI2, SI1). For comparison, we used the velocity values after the impact calculated from equation conservation of dynamics. After that is possible to analytically calculated velocities of bodies after the impact (collision) whereas for particular $e$ have equations form

$$
w_{1}=\frac{m_{2} v_{2}(1+e)+v_{1}\left(m_{1}-e m_{2}\right)}{m_{1}+m_{2}}, w_{2}=\frac{m_{2} v_{2}(1+e)+v_{1}\left(m_{1}-e m_{2}\right)}{m_{1}+m_{2}} .
$$

The analysis was carried out on basis of several simulations in the choice of various types of integrators, i.e. WSTIFF, GSTIFF, and HASTIFF. Based on the sensitivity analysis was selected method SI1 in which solver algorithms monitor the deviation for the relocation, for the velocity and for the Lagrange coefficient and for the generalized momentum of dynamics and the acceleration. This means that this method is the most accurate of these methods, however, calculation takes a lot of time. Series of dynamic simulations were performed with the calculation step-size of 0.00002 with coefficient of restitution $e=1$ and the penalty coefficient of $1,5 e+006$. From the (Fig. 8), it is clear that the most accurate results are observed at the choice of the integrator WSTIFF. The calculated values by (Eq. 3) are $w_{1}=6.3636 \mathrm{~m} / \mathrm{s}, w_{2}=10.3636 \mathrm{~m} / \mathrm{s}$ and the values received from MSC.ADAMS are exactly the same (Fig. 8).

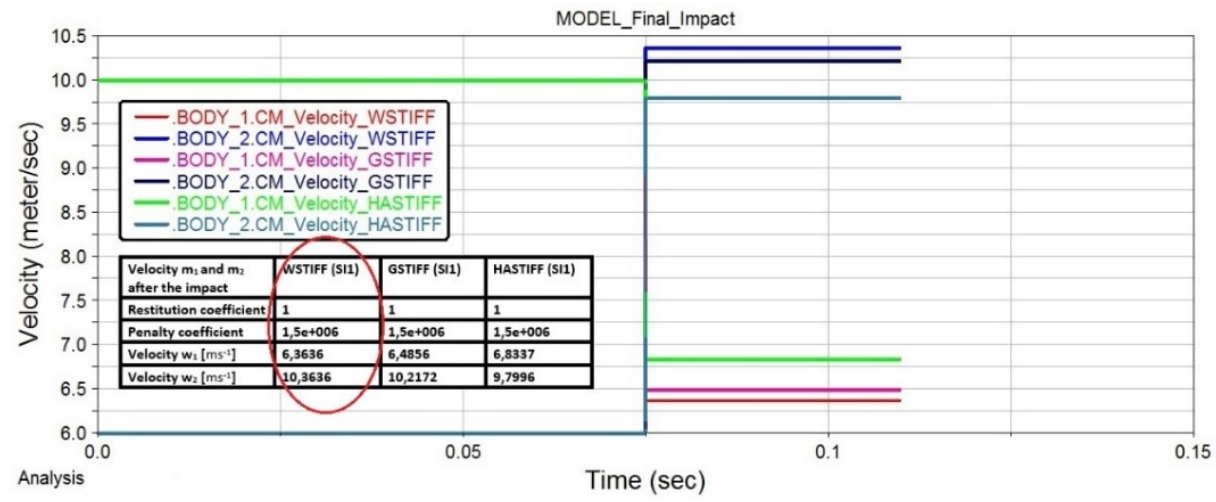

Fig. 8. The comparison of accurate particular solvers

\subsection{Influence of penalty coefficient}

In an environment of software, MSC.ADAMS is represented as an intermittent direct impact coefficient of restitution in addition to defining a penalty coefficient. Its task is to define an attribute stiffness between the contact materials. The value of the penalty parameter is ensured, that the penetration of one geometry to another is small. However, the large values can cause difficulties in numerical integration $[1,4,8]$. Creators of the set value $1 \mathrm{E} 6$ is suitable for systems modeled in $\mathrm{kg}-\mathrm{mm}-\mathrm{sec}$. We issue from the theoretical background that by the flexible impact $(e=1)$ is valid equation conservation of dynamics and equation conservation of mechanical energy. Were performed sensitivity analysis where the input variables are the coefficients of restitution and penalty coefficient (analyzed for each ,,e“), the target function was to ensure the compliance of the equation conservation of mechanical energy. The sum of the velocities of bodies by $e=1$, before the 
impact is equal to the amount after the impact. (Fig. 9) displays the progress of the impact force by perfectly elastic, plastic and perfectly plastic bodies. (Fig. 10) shows the velocity by significant coefficients $e$. By inelastic impact $(0 \leq e \leq 1)$ is valid only equation conservation of momentum. During the simulations when $e=0$ is monitored velocity in both bodies after the impact the same $\left(w_{1}=w_{2}\right)$. Fig. 11 is illustrated the process of translational kinetic energy by the same input parameters as by solving the velocity.

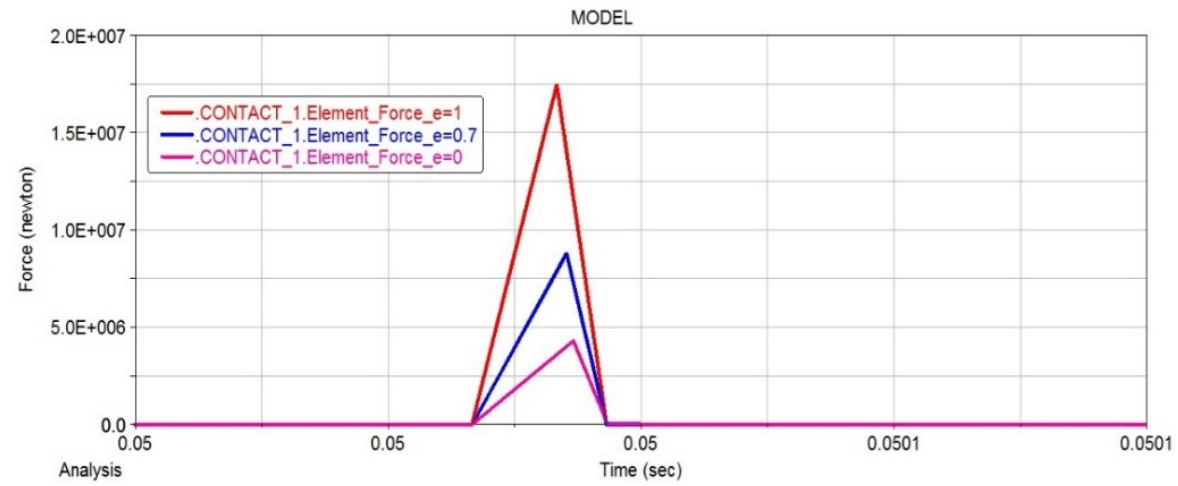

Fig. 9. Graphical representation of the contact force by different coefficients of resitution

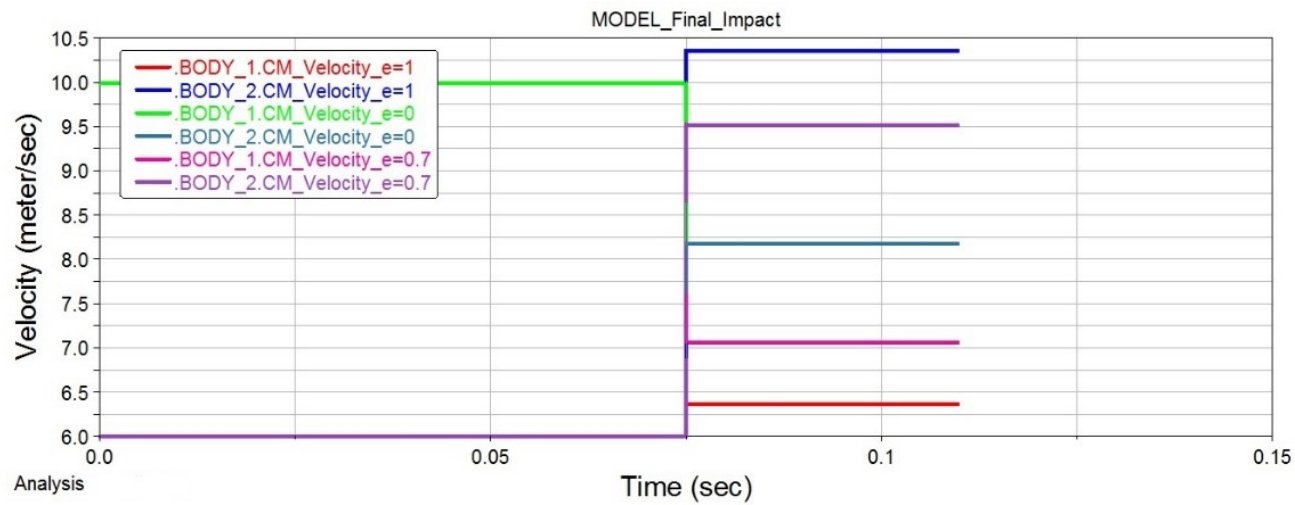

Fig. 10. Graphical representation of velocities both bodies by different coefficients of restitution

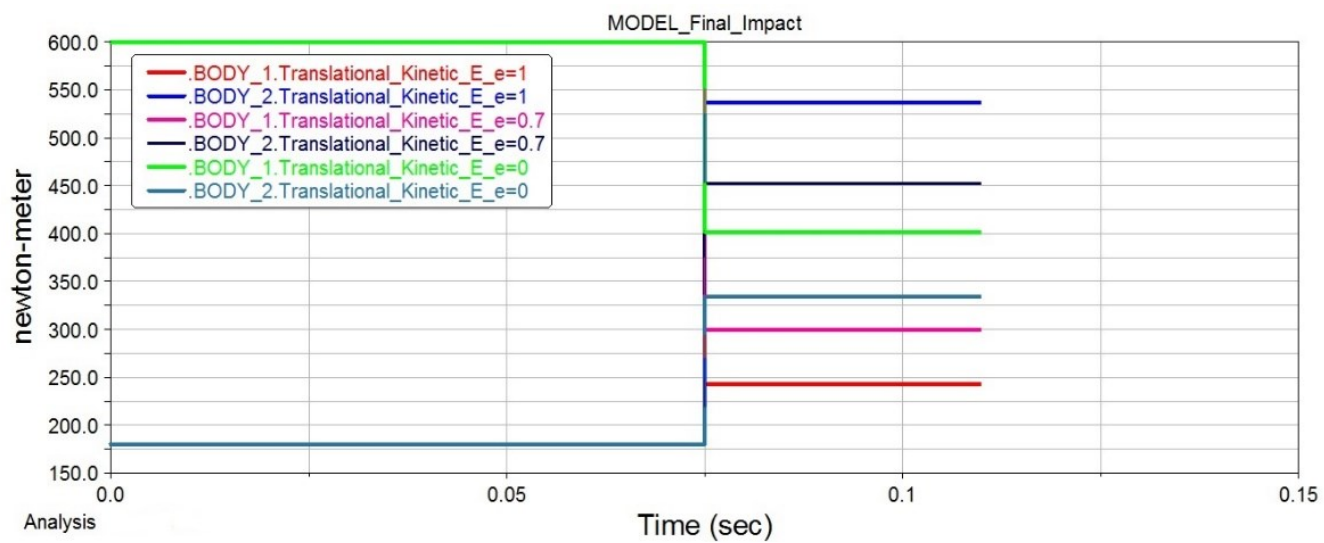

Fig. 11. Graphical representation of kinetic energy both bodies by different coefficients of restitution 
From process of (Fig. 8-10) follow, that the selected parameters of solver correspond and the results are consistent with the reality. With the default parameters of solution algorithms was made as row of simulation with different coefficients of restitution and the results were verified with numerical results (Eq. 3). This approach is documented in (Table 2).

Table 2. Influence of restitution coefficient and penalty coefficient

\begin{tabular}{|c|c|c|c|c|c|c|}
\hline WSTIFF, SI1 & \multicolumn{2}{|c|}{ No. 1} & \multicolumn{2}{|c|}{ No. 2} & \multicolumn{2}{|c|}{ No. 3} \\
\hline $\begin{array}{ll}\text { Coefficient } & \text { of } \\
\text { Restitution } & \end{array}$ & \multicolumn{2}{|r|}{1} & \multicolumn{2}{|c|}{0,7} & \multicolumn{2}{|r|}{0} \\
\hline Penalty coefficient & \multicolumn{2}{|c|}{$1,5 \mathrm{e}+006$} & \multicolumn{2}{|c|}{$1,5 \mathrm{e}+007$} & \multicolumn{2}{|c|}{$1,5 \mathrm{e}+008$} \\
\hline & Analyt. & ADAMS & Analyt. & ADAMS & Analyt. & ADAMS \\
\hline Velocity $w_{1}\left[\mathrm{~ms}^{-1}\right]$ & 6,3636 & 6,3636 & 6,9091 & 7,069 & 8,1818 & 8,1819 \\
\hline Velocity $\mathrm{w}_{\mathbf{2}}\left[\mathrm{ms}^{-1}\right]$ & 10,363 & 10,3636 & 9,7091 & 9,5172 & 8,1818 & 8,1819 \\
\hline $\begin{array}{l}\text { Kinetic energy before } \\
\text { the IMPACT } \mathrm{m}_{1}- \\
\text { EK }_{1}\end{array}$ & 600 & 600 & 600 & 600 & 600 & 600 \\
\hline $\begin{array}{l}\text { Kinetic energy before } \\
\text { the IMPACT } \mathrm{m}_{2}- \\
\mathrm{EK}_{2}\end{array}$ & 180 & 180 & 180 & 180 & 180 & 180 \\
\hline $\begin{array}{l}\text { Kinetic energy after } \\
\text { the IMPACT } \text { m }_{1}- \\
\text { EK }_{1}{ }^{*}\end{array}$ & - & 242,975 & - & 299,821 & - & 401,698 \\
\hline $\begin{array}{l}\text { Kinetic energy after } \\
\text { the IMPACT } \mathrm{m}_{2}- \\
\text { EK }_{2}{ }^{*}\end{array}$ & - & 537,024 & - & 452,889 & & 332,156 \\
\hline $\begin{array}{l}\text { The fulfillment of the } \\
\text { condition of kinetic } \\
\text { energy before } \\
\mathrm{EK}=\left(\mathrm{EK} \mathrm{K}_{1}+\mathrm{E} \mathrm{K}_{2}\right) \text { and } \\
\text { after } \\
\mathrm{EK}^{*}=\left(\mathrm{EK}_{1}{ }^{*}+\mathrm{EK} \mathrm{K}_{2}{ }^{*}\right)\end{array}$ & - & $\begin{array}{c}\mathrm{EK}=\mathrm{EK}^{*} \\
780=779,99\end{array}$ & - & $\begin{array}{c}\mathrm{EK}>\mathrm{EK}^{*} \\
780>752,71\end{array}$ & - & $\begin{array}{c}\mathrm{EK} \gg>\mathrm{EK}^{*} \\
780 \gg>733,84\end{array}$ \\
\hline $\begin{array}{l}\text { Maximum contact } \\
\text { force }[N]\end{array}$ & - & $5,4289 \mathrm{e}+6$ & - & $5,19 \mathrm{e}+8$ & - & $5,7022 \mathrm{e}+6$ \\
\hline
\end{tabular}

From the comparison of the obtained contact force by the different coefficients of restitution was confirmed, that the the bodies are more plastic if the force is lower. From the (Tab. 3) and from (Fig. 8-11) is validated the theoretical background and suitability of obtained parameters of sensitivity analysis by solving the velocity, kinetic energy and impact force in progress of function IMPACT.

\section{Conclusion}

In the previous sensitivity analysis, we have seen the significant influence of input contact parameters of the impact force process, i.e., selection of suitable time steps, restitution coefficient and coefficient of penalization. An important aspect is also the correct choice of the integrator for DAE equations solution. The accuracy and precision of simulation results should always be verified by comparing the results with the solutions of equations in closed shape (if obtainable) or the monitoring of the total energy.

The part of the results of this work has been supported by VEGA grant No. 1/0795/16. 


\section{References}

1. A. Sapietová, L. Gajdoš, V. Dekýš, M. Sapieta, Analysis of the Influence of Input Function Contact Parameters of the Impact Force Process in the MSC. ADAMS, ADVANCED MECHATRONICS SOLUTIONS Book Series: Advances in Intelligent Systems and Computing 393, 243-253 (2016)

2. M. Handrik, P. Kopas, V. Baniari, M. Vaško, M. Sága, Analysis of stress and strain of fatigue specimens localised in the cross-sectional area of the gauge section testing on bi-axial fatigue machine loaded in the high-cycle fatigue region. Procedia Engineering 177, 516-519 (2017)

3. M. Žmindák, Z. Pelagič, M. Nagel, L. Smetanka, Z. Stankovičová, Simulation modeling of barrel bore wear under dynamic pressure load. Communications 17 (3), 51-56 (2015)

4. A. Sapietová, M. Sapieta, B. Hyben, Sensitivity Analysis Application for Multibody System Synthesis. Applied Mechanics and Materials 420, 68-73 (2013)

5. K. Johnson, Contact Mechanics. (Cambridge University Press, Cambridge, 2013)

6. J. Giesbers, Contact Mechanics in MSC Adams - A technical evaluation of the contact models in multibody dynamics software MSC Adams. [Bachelor Thesis], University of Twente, 2013

7. M. Sága, M. Žmindák, V. Dekýš, A. Sapietová, Š. Segl’a, Selected methods of analysis and synthesis of mechanical systems. (VTS at University of Žilina, 360 p., 2009)

8. L. Jakubovičová, M. Sága, Computational analysis of contact stress distribution in the case of mutual stewing of roller bearing rings. Novel Trends in Production Devices and Systems, Applied Mechanics and Materials 474, 363-368 (2014)

9. V. Dekýš, A. Sapietová, O. Števka, Understanding of the dynamical properties of machines based on the interpretation of spectral measurements and FRF. Experimental stress analysis, Applied Mechanics and Materials 486, 106-112 (2014) 